\title{
Railway track availability modelling with opportunistic maintenance practice
}

\author{
Ben Davies and John Andrews
}

\begin{abstract}
The geometry of railway track must be maintained within certain standards in order to provide a highly available network, as well as good ride comfort and safety for all rail users. Modelling is well utilised as part of asset management tools in exploring the effectiveness of different rail geometry maintenance strategies. By considering the rail route as an entire system - in contrast to a track section in isolation - a more effective maintenance strategy can be developed, including the deployment of opportunistic maintenance practice. This study presents a Coloured Petri Net model of railway track degradation, inspection, and maintenance planning and delivery, for an entire route of track sections. Opportunistic maintenance is introduced through a novel search transition function, which groups interventions based on local adjacency. Testing explores the availability and maintenance demands expected when following a series of different management strategies. This is extended to testing under heatwave conditions, a known disrupter to track geometry maintenance delivery. Simulation results show that in following an opportunistic strategy, greater availability can be achieved on the modelled rail line. Further, resilience to the heatwave disruptions can be achieved by selecting the correct maintenance strategy parameters. This asset management tool can provide guidance on management strategies for a full route of track sections as a combined system.
\end{abstract}

\section{Keywords}

Climate change, Coloured Petri net model, Degradation model, Heatwave, Opportunistic maintenance, Railway track degradation, Tamping, Track geometry degradation

\section{Introduction}

The UK rail network comprises of more than 20,000 miles of track. As utilisation is forecast to continue growing, there is increasing demand for effective delivery of maintenance activities. To support this, a tool is described which assesses the suitability of different maintenance strategies. Of particular concern is the need to control the geometry of the track. This paper builds on a previous iteration of the railway track asset management tool presented in Davies and Andrews $^{1}$. The former model is a Petri net model, where-in a generic track section is simulated. However, informed decisions can be made when considering the greater system, i.e., whole route performance. By introducing interdependency between multiple track sections, opportunistic maintenance can be delivered. This is facilitated in using a Coloured Petri Net (CPN) approach.

\section{Track geometry and maintenance}

Maintaining railway track geometry within certain limits is vital to provide safe and comfortable journeys for all users. Traffic loading will however lead to geometry degradation, as the track support ballast settles or suffers wear and breakage ${ }^{2}$. Maintenance actions re-pack or replace the ballast material, supporting the track sleepers and restoring the rail running surface to the required position. Rail asset management strategies typically employ a combination of corrective (repairing a faulty condition) and preventive (acting before major problems develop) maintenance approaches ${ }^{3}$.

Rail asset management begins with establishing the condition of the track geometry. This is measured in three dimensions by an instrumented Track Recording Unit (TRU), which periodically inspects rail sections as it travels around the network. Of the measurement parameters available, the standard deviation of the vertical profile over a $35 \mathrm{~m}$ length is considered by many to be the most significant indicator of track geometry condition $^{4-7}$. This measure shows good correlation with the vehicle ride quality and is readily recovered using ballast maintenance activities ${ }^{8}$.

Good track condition encompasses geometry measures close to the designed profile. This provides good ride comfort and safety, and allows the network to be used at full traffic capacity. An extremely bad track condition, in contrast, has geometry very far from that desired, and carries a significant safety risk for vehicle derailment ${ }^{9}$. This major degradation condition would warrant restricting the traffic use of the rail line, and demand corrective maintenance to quickly restore the geometry and network availability.

Between these extremes, a minor degradation would define a poor track condition, with some small

\footnotetext{
Resilience Engineering Research Group, University of Nottingham, UK

Email: Ben.Davies@nottingham.ac.uk
} 
deviation from the required vertical geometry. Network availability may not be affected if the rail section can still be used to capacity, but a preventive maintenance action would be scheduled. This work should repair the geometry to the good condition before a major degradation develops. The goal is to provide continuous availability through the system lifetime, and prevent disruption. Andrade and Teixeira ${ }^{10}$ present a simple model calculation which reveals a strong correlation that increasing maintenance delivery decreases network delays. Track asset management should be motivated to deliver efficient maintenance schedules in order to avoid delay penalties ${ }^{11}$.

When a degraded condition is detected, the asset management process proceeds in selecting an appropriate intervention activity. For ballast maintenance, the most common option is tamping ${ }^{12}$ with stoneblowing emerging as a modern alternative ${ }^{13}$. Both approaches lift the rails into the correct geometry position; tamping machines then repack ballast around the sleepers, whilst stoneblowers fill the void with stone chips. Asset management policy must select the correct maintenance activity to be employed for the local rail condition.

Tamping is however an involved process that can add to the physical damage of the ballast material and lead to increased geometry degradation rates thereafter ${ }^{7}$. Successive tamping operations will therefore reduce in effectiveness, with management strategies typically defining a limit before ballast renewal ${ }^{14}$.

Stoneblowing does not disturb the existing, compacted ballast bed. This can provide longer lasting results than the equivalent tamping action. However, once the small stone chips have been added to the track support, tamping should not be performed thereafter, as the smaller material can cause secondary problems such as blocking drainage of the track bed ${ }^{7,15}$.

Maintenance scheduling practice With the goal of sustaining route availability, the final task of the rail asset management strategy is to schedule the completion of the maintenance works. As has been identified, a major degradation would necessitate corrective maintenance, to restore the track geometry. This is scheduled urgently, to be carried out very soon after discovery. In order to minimise the duration of any traffic restrictions, this could be within a single day ${ }^{13}$.

A minor geometry degradation, in contrast, requires preventive maintenance to ensure the continued upkeep of the track condition. This is scheduled as routine maintenance, to fit in with the availability of rail operations, equipment, and personnel. Routine maintenance should not restrict route availability itself, and so is completed overnight, out of operating hours. The routine schedule will be some time after the geometry inspection, perhaps as much as three months ${ }^{4}$.

Opportunistic maintenance (OM) is an extended feature of a preventive maintenance strategy, which allows restorative works to be completed in advance of the planned schedule. Such an opportunity becomes available when a process is halted - when outside of operating hours, carrying out required maintenance, or potentially the result of a failure - and multiple works can be completed at the same time. The core objective is to complete as much maintenance as is efficient within the time window of the stoppage ${ }^{16}$.

Advantages are found by reducing the number and frequency of process suspensions for maintenance, as well as efficiently planning the availability of equipment, materials, and personnel ${ }^{17}$. OM is particularly useful in systems with a high availability demand, and a high cost associated with down-time ${ }^{18}$. The rail network is a system which fits these criteria ${ }^{19}$.

With respect to track geometry maintenance, windows of opportunity become available when a rail line possession takes place to complete works ${ }^{20}$. Other maintenance activities may be brought forward in their own schedule to be completed as a single work order during the same possession event. Opportunity therefore arises with a combination of neighbouring location and similar maintenance activity ${ }^{20}$. Literature sources exploring OM approaches to rail management tend to follow one of two classes; predetermined asset groups, or pre-calculated maintenance requirements.

Firstly, models which take defined groups of assets as an input, and seek to optimise the group size for maximum maintenance effect. Simson et al. ${ }^{21}$ model the condition of sleepers in up to nine adjacent track sections, implemented using a series of linked EXCEL spreadsheets. Dell'Orco et al. ${ }^{22}$ input clusters of track sections ranging from two to seven, with a fuzzy inference system deciding the tamping schedule. Oyama and Miwa ${ }^{23}$ group track sections into "units" of between three and ten, also for a unified tamping schedule. There is however limited scope for extending these approaches to the wider network, where a vast number of asset groups must be predefined and managed. Santos et al. ${ }^{24}$ are able to mitigate this limitation through efficient decision making algorithms, though this is only tested for a one-year schedule horizon.

The second group of studies optimise the cost implications of grouping similar maintenance activities from an expected schedule. Famurewa et al. ${ }^{25}$, Zhang et al. ${ }^{20}$, and Khajehei et al. ${ }^{26}$ perform optimisation on a predicted volume of maintenance. Optimisation constraints are defined for minimising set-up costs (of a single maintenance action) and the expense of the remaining-useful-life that is lost due to an early intervention. Zhao et al. ${ }^{27}$ goes further in modelling three different renewal activities, and forming optimal groups of both geographical location and maintenance type. Peng et al. ${ }^{28}$ pursues a similar optimisation goal, to schedule activities to minimise the travel distances between each project. These methods are to be applied in planning a management strategy, where the time to maintenance is known, and are not agile enough to consider shorter-term activities.

Petri net (PN) modelling has been demonstrated to be a highly flexible method for several successful asset management studies ${ }^{1,13,29-32}$. Of these, 
Prescott and Andrews present the most complete exploration of railway track geometry management with opportunistic maintenance ${ }^{13}$. This approach includes modules which make network-level decisions based on the number and type of maintenance activities demanded in geographical regions, as well as modelling the availability of a limited number of maintenance machines - both for tamping and stoneblowing. One weakness of this model is that the rail degradation module would, by necessity, be repeated for each track section across the network, leading to a unwieldy model with much duplication.

Influence of hot weather The completion of maintenance works can be subject to other external factors beyond the availability of engineering teams. One such factor is hot weather ${ }^{9}$. Elevated summer temperatures cause the rail to expand longitudinally against its fixings, with a risk of buckling if not correctly supported ${ }^{33}$. Both tamping and stoneblowing activities are suspended when rail temperatures exceed a $32{ }^{\circ} \mathrm{C}$ limit $^{9}$, as lifting the track into position would offer the freedom to buckle. This would result in a greater maintenance problem than was originally existed.

There are few existing studies of asset management practice that consider the effects of hot weather. It is estimated the heat-related delays cost UK rail managers between $£ 3 m$ and $£ 15 \mathrm{~m}$ annually during modern summers, and predict up to $£ 23 \mathrm{~m}$ annually in 60 years time under climate change projections ${ }^{34}$. Dobney et al. conclude that "ensuring the track is thoroughly maintained would reduce the vulnerability of the rail during hot weather" ${ }^{19}$. The influence of high temperatures was first explored as an inhibiting factor in the asset management model in Davies and Andrews ${ }^{1}$. This model studies track sections in isolation, which is now expanded to consider the interdependencies between all sections which comprise a rail line.

This paper contributes a Coloured Petri Net model of railway track geometry degradation and management, with opportunistic maintenance included in the scheduling strategy. The CPN capitalises on the strengths of the conventional Petri net models for this area of study, in a compact format graphical model. A novel search transition is introduced, for the delivery of opportunistic maintenance practice.

\section{Track Asset Management Model}

\section{Coloured Petri Net Modelling}

Coloured Petri nets are a high-level extension of the conventional Petri net. This increases complexity in the tokens. With conventional PNs there is only one kind of token, representing the Boolean state of a place (either marked or unmarked). In the high-level net, a coloured token can carry complex information about the system or process, and are distinguishable from one-another. The following description shall assume familiarity with the structure and function of a standard Petri net, and therefore focus on the capabilities afforded by the addition of colours. a)
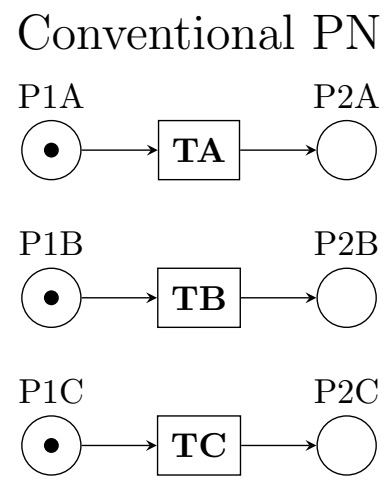

b)

\section{Coloured PN}

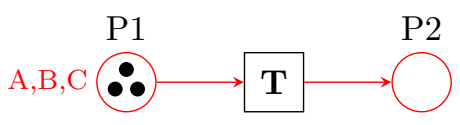

Colour ID $=\{\mathrm{A}, \mathrm{B}, \mathrm{C}\}$

!

Figure 1. Example of the compact Coloured Petri Net model

\section{Colours and colour sets}

Colours and colour sets are analogous to programming languages with elaborate type definitions ${ }^{35}$. Colours are the data values assigned to the tokens. These may be of arbitrarily complex type, such as an integer number, a text string, or a list of records. A colour set is assigned to a given place holding these coloured tokens. All tokens in the place must be of the same type, matching the colour set. An example is a colour set of integer ID numbers, where each token is assigned a numeric identifier.

Thus the compact CPN approach can represent multiple assets, simultaneously following the same system processes. An example $\mathrm{PN}$ containing three similar modules A, B, and C is illustrated in figure 1.a). These are combined into a single CPN module, figure 1.b). The colour set is declared in the dashed box, with a character set for the identity of each asset. Place $P 1$ contains three uniquely coloured tokens, as indicated by the labelling, representing the different assets.

Colour definitions go beyond unique asset identifiers, carrying such information as the type, location, and maintenance history of an asset. By reading the colour values in the input tokens, unique transition behaviours can also be affected. Internal transition functions can handle simulation timing including conditional relations, periodic transitions, routing, resetting, and the novel search transition, as discussed in the following sections. When properly defined, transition $T$ in the compact $\mathrm{CPN}$ example in figure 1.b) can enact different transition processes for each token identity $A, B, C$, or localised system asset. 
a) Initially

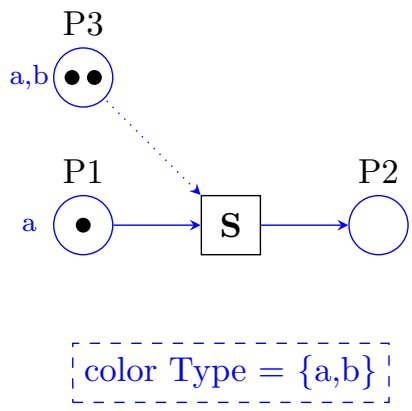

Search function: match Type

b)

\section{After firing}

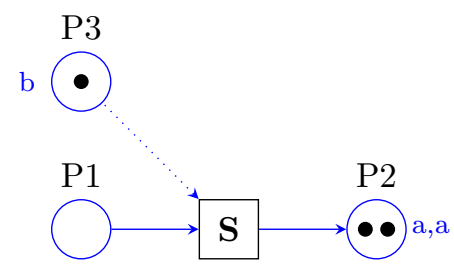

Figure 2. Search transition function in the CPN

\section{Time in the CPN}

The uncoloured PN typically handles time within the transitions, in the form of a firing delay. In the CPN, this time delay is moved to the tokens, with an timestamp integer colour set. This describes the number of simulation iterations that must pass before a token is considered "ready" to fire, i.e., it has completed the current system process. Transitions are eager to fire, as soon as the input tokens are available. Functions within the transition blocks assign the token timestamps, with the same variety as in the conventional PN; instant, fixed time, or stochastically sampled.

Following the example in figure 1, even if transition times $T A, T B$, and $T C$ are different, the CPN transition $T$ can consult the input token identities to assign delays as appropriate.

\section{Search transition}

The search transition is a new development for this CPN model. When this transition fires, the search function acts to find other tokens within prescribed places which satisfy the search criteria. In this approach, token movements can be controlled by the states of other tokens in the net. An example is provided in figure 2.

In this example, the search transition $S$ accepts an input token from place $P 1$ with an asset type colour value of $a$. The search arc connects to place $P 3$, which contains two unique tokens with asset colour values of $a$ and $b$ respectively. The search function finds tokens which match the type value of the input token. Thus, after firing, two tokens of type $a$ are moved to place $P 2$, whilst the type $b$ token remains in place $P 3$.
It is this functionality that facilitates OM in the rail geometry model; searching for maintenance demands with criteria for neighbouring location and similar activity type.

\section{Route geometry model structure}

The Coloured Petri Net model presented herein is designed to provide a flexible and easily managed tool for testing different track asset maintenance strategies. The model simulates the track geometry degradation, inspection, and maintenance of a 20 mile rail line, constructed of a sequence of 160 individual oneeighth mile (approximately $200 \mathrm{~m}$ ) track section assets. The results give a prediction of the availability of the rail line, the incidence of traffic disruptions, and the volume of maintenance work expected when following a given strategy. As presented, the model is parameterised for a rail line of the greatest required geometry quality, where vehicle speeds are in excess of $100 \mathrm{mph}$. This can be rapidly reconfigured to simulate the maintenance of a different quality rail line, or indeed any asset system of comparable management process, by defining the appropriate degradation rates and maintenance strategy.

Figure 3 shows the structure of the CPN model, with the initial marking at the start of the lifetime simulations. The places are labelled to indicate the different conditions of the track sections, and the management processes. The transitions are coded: D for degradation; I for inspection; P for policy; $\mathrm{M}$ for maintenance scheduling; $\mathrm{S}$ for search; $\mathrm{C}$ for maintenance completion; $\mathrm{W}$ for weather. The following describes the modular structure, with reference to the CPN elements in the figure.

Colour set definitions Three colour sets are included in the CPN model, and are summarised in table 1 . The first represents the track assets. Each asset has a unique alphanumeric identification; a sequential numeric record of the location in the route; a numeric count for the history of maintenance interventions, recording tamping and stoneblowing separately; and the timestamp for transition firing. The knowledge colour set represents the information captured by the asset management process. This colour set carries the same data as the asset tokens, adding a value for the condition at the point of inspection. The distinction between these two colour sets is that the former is the unknown state of the track in-situ, and the latter is the information that asset management act upon. Finally, the basic colour set contains no attributes beyond the transition timestamp; this is similar in function to the conventional PN token, and simply denotes the Boolean marking of the weather places.

In the visual representation of the CPN model, figure 3, the elements are colourised to denote which colour set is assigned; red for the track assets, in the top row of the figure; blue for the knowledge set; and black for the basic set only related to the weather cycle. 


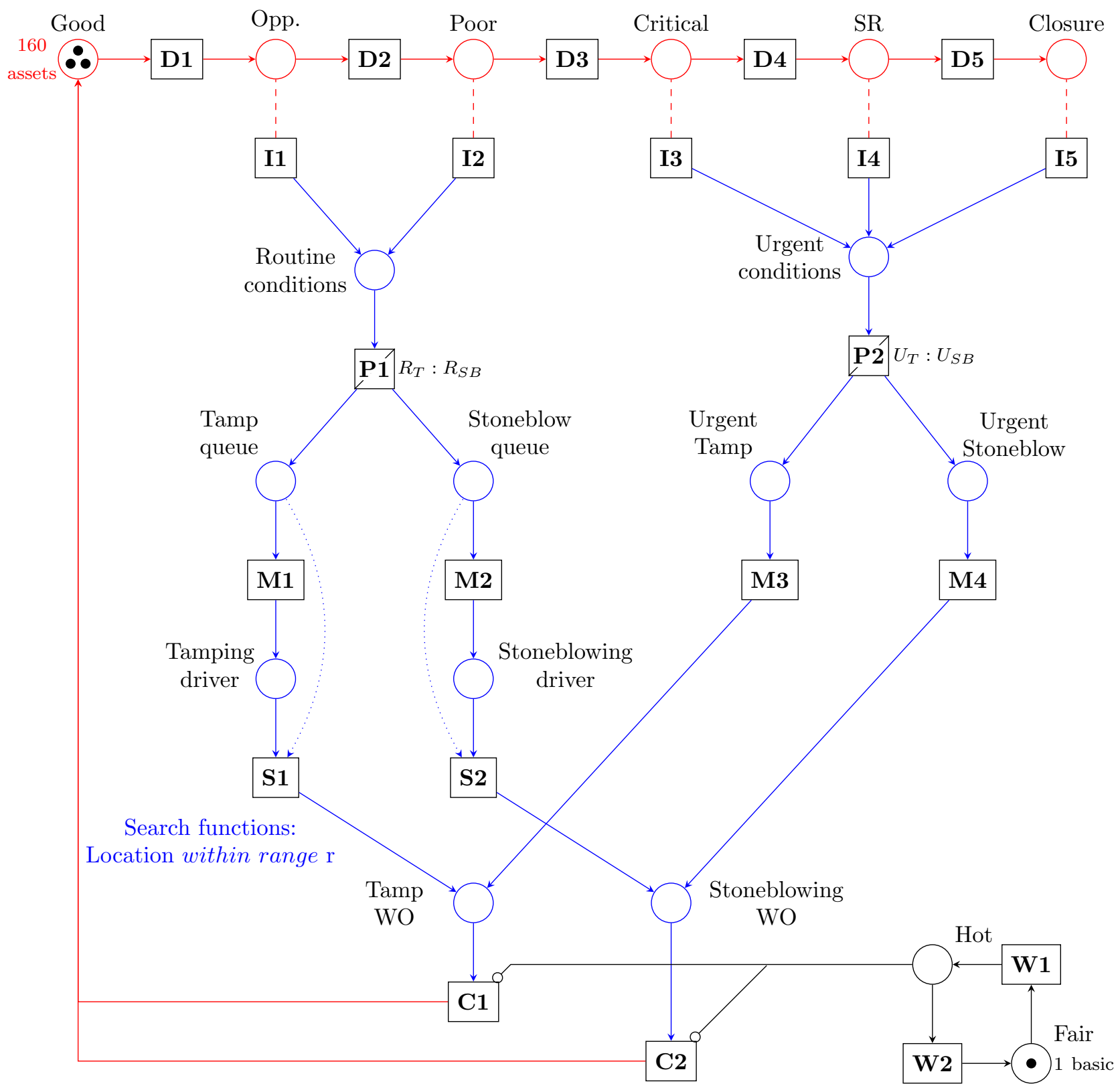

Figure 3. Coloured Petri Net model of track route degradation, inspection, and maintenance

Table 1. CPN model colour set declaration

\begin{tabular}{|c|c|c|}
\hline $\begin{array}{r}\text { colour set asset: } \\
\text { section ID } \\
\text { location } \\
\text { tamp history } \\
\text { stoneblow history } \\
\text { timestamp }\end{array}$ & $\begin{array}{l}= \\
= \\
= \\
=\end{array}$ & $\begin{array}{l}\text { string } \\
\text { integer } \\
\text { integer } \\
\text { integer } \\
\text { integer }\end{array}$ \\
\hline $\begin{array}{r}\text { colour set knowledge: } \\
\text { section ID } \\
\text { location } \\
\text { condition } \\
\text { tamp history } \\
\text { stoneblow history } \\
\text { timestamp }\end{array}$ & $\begin{array}{l}= \\
= \\
= \\
= \\
= \\
=\end{array}$ & $\begin{array}{l}\text { string } \\
\text { integer } \\
\{\mathrm{O}, \mathrm{P}, \mathrm{C}, \mathrm{S}, \mathrm{L}\} \\
\text { integer } \\
\text { integer } \\
\text { integer }\end{array}$ \\
\hline $\begin{array}{r}\text { colour set basic: } \\
\text { timestamp }\end{array}$ & $=$ & integer \\
\hline
\end{tabular}

Degradation process At the top of figure 3 are places for each of the track section condition states and the degradation transitions between them, $D 1-5$. The six condition states are defined by thresholds in the measurable vertical geometry deviation: good is close to the desired geometry; the opportunistic threshold $\sigma_{o p p}$ is a satisfactory condition where OM may begin; the poor condition threshold, $\sigma_{\text {poor }}$, is a minor degradation state where traffic continues to pass at full capacity, and routine maintenance is scheduled; the critical condition uses threshold $\sigma_{\text {crit }}$, close to the major degradation state though without traffic restrictions, but requiring urgent maintenance to prevent future disruption; $\sigma_{S R}$ is the first major degradation threshold, wherein a traffic speed restriction should be enforced; and $\sigma_{\text {close }}$ is the major geometry degradation so great that a closure of the faulty line is required.

The geometry condition thresholds are defined as $\sigma_{\text {poor }}=1.9 \mathrm{~mm}, \sigma_{\text {crit }}=2.7 \mathrm{~mm}, \sigma_{S R}=3.4 \mathrm{~mm}$, and $\sigma_{\text {close }}=5.0 \mathrm{~mm}$. A previous study found these are good values for the minor condition thresholds, poor and 
Table 2. Weibull parameters used in the degradation transitions

\begin{tabular}{|c|c|c|c|c|c|c|c|c|}
\hline $\begin{array}{l}\text { Number } \\
\text { of tamps }\end{array}$ & $\begin{array}{l}D 10.0- \\
\eta \text { (days) }\end{array}$ & $\begin{array}{l}.9 \mathrm{~mm} \\
\beta\end{array}$ & $\begin{array}{l}D 31.9- \\
\eta \text { (days) }\end{array}$ & $.7 \mathrm{~mm}$ & $\begin{array}{r}\text { c } D 42.7 \\
\eta \text { (days) }\end{array}$ & $\begin{array}{l}3.4 \mathrm{~mm} \\
\beta\end{array}$ & $\begin{array}{l}D 53.4- \\
\eta \text { (days) }\end{array}$ & $\mathrm{mm}_{\beta}$ \\
\hline 0 & 2701.6 & 0.950 & 1044.3 & 0.299 & 4674.9 & 0.296 & 2240.1 & 0.372 \\
\hline 1 & 2224.3 & 0.996 & 2222.3 & 0.351 & 3653.7 & 0.311 & 3542.0 & 0.361 \\
\hline 2 & 2199.8 & 1.108 & 10105.0 & 0.316 & 3008.7 & 0.308 & 1116.4 & 0.455 \\
\hline 3 & 2080.0 & 1.147 & 7947.2 & 0.310 & 2363.6 & 0.314 & 1679.7 & 0.426 \\
\hline 4 & 2025.0 & 1.195 & 11767.0 & 0.312 & 2240.1 & 0.311 & 1352.2 & 0.447 \\
\hline 5 & 1970.0 & 1.245 & 6932.3 & 0.299 & 2497.8 & 0.305 & 1746.6 & 0.438 \\
\hline 6 & 1915.0 & 1.295 & 14602.0 & 0.273 & 2967.5 & 0.29 & 1733.6 & 0.441 \\
\hline 7 & 1770.0 & 1.390 & 35174.0 & 0.273 & 9682.6 & 0.264 & 1449.0 & 0.419 \\
\hline $\begin{array}{l}\text { Number } \\
\text { of tamps }\end{array}$ & \multicolumn{2}{|c|}{$\begin{array}{l}D 10.0-0.5 \mathrm{~mm} \\
\eta \text { (days) } \quad \beta\end{array}$} & \multicolumn{2}{|c|}{$\begin{array}{l}D 10.0-1.0 \mathrm{~mm} \\
\eta \text { (days) } \beta\end{array}$} & \multicolumn{2}{|c|}{$\begin{array}{l}D 10.0-1.5 \mathrm{~mm} \\
\eta \text { (days) } \beta\end{array}$} & & \\
\hline 0 & 2388.0 & 0.810 & 2500.0 & 0.860 & 2612.0 & 0.910 & & \\
\hline 1 & 2186.5 & 0.940 & 2200.0 & 0.960 & 2213.5 & 0.980 & & \\
\hline 2 & 1889.0 & 0.940 & 2000.0 & 1.000 & 2111.0 & 1.060 & & \\
\hline 3 & 1800.0 & 1.105 & 1900.0 & 1.120 & 2000.0 & 1.135 & & \\
\hline 4 & 1675.0 & 1.125 & 1800.0 & 1.150 & 1925.0 & 1.175 & & \\
\hline 5 & 1550.0 & 1.175 & 1700.0 & 1.200 & 1850.0 & 1.225 & & \\
\hline 6 & 1425.0 & 1.225 & 1600.0 & 1.250 & 1775.0 & 1.275 & & \\
\hline 7 & 1350.0 & 1.250 & 1500.0 & 1.300 & 1650.0 & 1.350 & & \\
\hline $\begin{array}{l}\text { Number } \\
\text { of tamps }\end{array}$ & \multicolumn{2}{|c|}{$\begin{array}{l}D 20.5-1.9 \mathrm{~mm} \\
\eta \text { (days) } \beta\end{array}$} & \multicolumn{2}{|c|}{$\begin{array}{l}D 21.0-1.9 \mathrm{~mm} \\
\eta \text { (days) } \beta\end{array}$} & \multicolumn{2}{|c|}{$\begin{array}{l}D 21.5-1.9 \mathrm{~mm} \\
\eta \text { (days) } \beta\end{array}$} & & \\
\hline 0 & 109.6 & 0.466 & 50.9 & 0.401 & 10.9 & 0.316 & & \\
\hline 1 & 103.3 & 0.336 & 41.4 & 0.297 & 6.8 & 0.244 & & \\
\hline 2 & 129.3 & 0.511 & 62.0 & 0.436 & 14.0 & 0.339 & & \\
\hline 3 & 124.3 & 0.492 & 71.0 & 0.448 & 24.9 & 0.384 & & \\
\hline 4 & 167.9 & 0.540 & 93.8 & 0.483 & 30.5 & 0.401 & & \\
\hline 5 & 225.4 & 0.586 & 130.4 & 0.525 & 45.0 & 0.433 & & \\
\hline 6 & 282.9 & 0.632 & 166.0 & 0.564 & 56.8 & 0.456 & & \\
\hline 7 & 223.2 & 0.626 & 115.7 & 0.526 & 31.2 & 0.403 & & \\
\hline
\end{tabular}

critical, for achieving high track section availability ${ }^{1}$. Furthermore, the values for the major degradation thresholds, speed restriction and closure, are required safety limits as defined in the standard for this rail class, and cannot be changed ${ }^{9}$.

Variant opportunistic maintenance strategies will test sensitivity to the opportunistic threshold. Three values are selected between the good and poor conditions; $\sigma_{\text {opp }}=0.5 \mathrm{~mm}, 1.0 \mathrm{~mm}$, or $1.5 \mathrm{~mm}$.

Degradation transitions $D 1-5$ dictate the progression from better to worse condition states. The transition functions provide stochastically sampled timestamps to the asset tokens following two-parameter Weibull probability distributions, with shape factor $\beta$, and characteristic lifetime $\eta$ in days. Weibull parameters were found to provide the best fit for empirical data; detail can be found in publications by Audley and Andrews ${ }^{7}$, and Andrews ${ }^{29}$. When the timestamp duration expires, the token is moved from transition source place to the target place, as dictated by the directed arrows. All token colour values remain the same except for the timestamp, which is reset and resampled by the subsequent transition function.

Table 2 lists the Weibull parameters used in $D 1-$ 5 , for the different condition threshold combinations. As has been discussed, track geometry degradation is found to be negatively effected by tamping actions. The degradation transition functions consult the number of actions in the asset token's tamping history colour set when sampling using the Weibull parameters. In this way, different distributions may be used for each asset, modelling the local degradation conditions for each rail section individually.

Inspection process The inspection transitions $-I 1-5$ - are periodic transitions which attempt to fire at regular intervals, independent of the timestamp on any input tokens. All are defined with an interval $\theta=$ 28 days, typical for rail lines of this class. For each token in a track condition place, the corresponding inspection transition generates a new knowledge token in the known condition places; opportunistic and poor conditions generate routine maintenance actions, critical, speed restriction, and closure conditions lead to urgent actions. This is the asset information gathered at the time of the inspection. The track tokens in the condition places remain, such that degradation process may continue.

Maintenance policy The policy transitions $P 1$ and $P 2$ are routing transitions, selecting a single output from the multiple connected places. These use probabilistic ratios to select one maintenance activity from the two options, either tamping or stoneblowing; $R_{T}: R_{S B}$ in $P 1$ for routine maintenance, $U_{T}: U_{S B}$ in $P 2$ for urgent actions. In practice, this is a decision made by asset managers based on factors such as the nature of the geometry fault, network maintenance targets, and equipment availability. It is however found to be well represented by a simple decision ratio, when considering an average for the national practice. 
The policy transition consults the knowledge token for the track condition and maintenance history to select the decision ratio to be used. In all cases, when there is a history of at least one stoneblowing activity tamping will no longer be available, with $R_{T}: R_{S B}=U_{T}: U_{S B}=0$ : 1 .

Whilst there is no history of stoneblowing in the token colour data, a ratio $R_{T}: R_{S B}=9: 1$ is followed for the opportunistic and poor conditions, and $U_{T}$ : $U_{S B}=3: 1$ for the critical, speed restriction, or closure conditions. Tamping is effective for a lesser geometry deviation condition, and highly preferred, whilst the proportion of stoneblowing actions increases in the major degradation conditions. Transitions $P 1$ and $P 2$ are otherwise untimed in the model, suggesting asset management takes immediate action on the knowledge gained in inspection; a time delay could be included if representative of the real-world practices.

Maintenance scheduling Knowledge tokens enter a scheduling queue dependant on their condition type and urgency. The maintenance schedule transitions M1 - 4 - introduce delay to the asset management process. This transition time makes account for the time for maintenance equipment to travel to the fault location when demanded.

A routine maintenance action is scheduled to fit in with existing operational demands across the rail network, and could be delayed for several months. This is however tolerable for a track section with measured opportunistic or poor condition, as traffic can continue to pass and network performance is not effected. In contrast, an urgent action is an emergency intervention which must be completed very soon after a major degradation is discovered, typically the same day.

These transitions follow stochastically sampled Normal distributions for delay times $N\left(\mu, \sigma^{2}\right)$, with mean response time $\mu$ and variance $\sigma^{2}$, both in days. For a poor condition knowledge token, $M 1$ and $M 2$ are sampled from $M_{\text {routine }}=N(90,15)$. Opportunistic condition knowledge tokens are not fired through these transitions and do not have time stamp values sampled. These tokens are only added to work orders when paired with a scheduled maintenance action; this is explored in the following section. For urgent maintenance, $M 3$ and $M 4$ are sampled from $M_{\text {urgent }}=N(0.5,0.5)$ in all cases.

Generating work orders A work order (WO) is the total volume of maintenance that is to be completed in a single possession event. Grouping maintenance actions together provides efficiency in the number and frequency of engineering possessions. Because of the disruptive nature of a major degradation condition, urgent maintenance is always completed as a single action WO; maintenance transitions $M 3$ and $M 4$ output directly to the appropriate WO places.

For routine maintenance, the first track section to come to schedule is considered the "driving" asset for the order of work. Search transitions $S 1$ and $S 2$ find any number of assets that require the same maintenance activity - either tamping or stoneblowing - within a given adjacency range $r$ expressed as a number of asset lengths. The internal search function compares each of the queued knowledge tokens with the location of the driving asset. In this way, multiple opportunistic and poor maintenance actions can be completed at once, saving the time and expense of multiple equipment setup and tear-downs, and increasing the overall work completion rate on the line.

In practice, an engineering team typically service a one mile length in a single possession ${ }^{36}$, with $r= \pm 5$ in either direction from the driving asset. Variant cases also compare longer two or five mile ranges, $r= \pm 9$ or \pm 21 . This is consistent with working speeds of up to $2 \mathrm{~km} / \mathrm{h}^{26}$.

Maintenance completion The final transitions $C 1$ and $C 2$ of the asset management model handle the delivery of the maintenance WO. On firing, all of the completed track sections are returned to the good condition, resetting asset markings in the other condition places. The relevant maintenance history colour value is incremented within the asset tokens. As the completion time is considered shorter than the one day simulation time step - a work shift is a matter of hours - these are untimed, instant transitions.

Temperature exceedances Hot weather is modelled as a simple loop between two states, fair or hot. A marking in the hot place represents a daily air temperature exceeding $21{ }^{\circ} \mathrm{C}$, or predicted to exceed $25{ }^{\circ} \mathrm{C}$ within three days. This would cause the rail temperatures to exceed $32{ }^{\circ} \mathrm{C}$ or $38^{\circ} \mathrm{C}$ respectively, the critical thresholds for rail management operators to suspend maintenance $\operatorname{activities}^{37}$. This definition considers only that the critical rail temperature is exceeded, not to what degree. As long as the heatwave persists maintenance activities must be postponed until the rails have cooled sufficiently, following the current policy.

Weather transitions $W 1$ and $W 2$ are respectively the time periods before a heatwave event occurs $\tau_{\text {fair }}$ and its duration $\tau_{\text {hot }}$. The delay $\tau_{\text {fair }}$ is used to ensure the system reaches a steady-state condition prior to the period of hot weather in all test scenarios. As long as the hot weather place is marked, maintenance completion transitions $C 1$ and $C 2$ are inhibited from firing. Although maintenance actions are due for completion, having completed their scheduling delay, the incidence of hot weather prevents their completion. This may lead to longer periods where speed restrictions and closures are enforced on the rail line, reducing overall availability for traffic flow. Whilst the weather disruption persists inspections will continue, and may add to the overall work order that must be completed once the heatwave ends.

Test scenarios follow a range of hot weather durations, with $\tau_{h o t}=1,2,3,4,5,10,15,20,25,30$ days. This will investigate the evolution of the track condition through a maintenance-free period, and how an opportunistic maintenance strategy can provide resilience to the rail line availability. 
Table 4. Non-OM strategy parameters

\begin{tabular}{ll}
\hline Parameter & Value \\
\hline Poor threshold & $\sigma_{\text {poor }}=1.9 \mathrm{~mm}$ \\
Critical threshold & $\sigma_{\text {crit }}=2.7 \mathrm{~mm}$ \\
Speed restriction threshold & $\sigma_{S R}=3.4 \mathrm{~mm}$ \\
Close threshold & $\sigma_{\text {close }}=5.0 \mathrm{~mm}$ \\
Inspection interval & $\theta=28$ days \\
Routine ratio & $R_{T}: R_{S B}=9: 1$ \\
Urgent ratio & $U_{T}: U_{S B}=3: 1$ \\
Routine completion rate & $F_{\text {routine }}=N(90,15)$ \\
Urgent completion rate & $F_{\text {urgent }}=N(0.5,0.5)$ \\
\hline
\end{tabular}

\section{Model execution}

Simulation of the CPN model follows a Monte Carlo approach, with random sampling for the transition processes which follow probabilistic distributions. The model uses a discrete time series; one-day per iteration. The model is tested firstly for a strategy without OM, following only the parameter values listed in table 4 - transition D2 instantly fires to the poor condition. Thereafter, OM is tested with nine combinations of opportunistic condition thresholds and search ranges; $\sigma_{\text {opp }}=0.5 \mathrm{~mm}, 1.0 \mathrm{~mm}$, or $1.5 \mathrm{~mm}$; and $r= \pm 5, \pm 9$, or \pm 21 .

The primary performance indicator is the rail line availability; when all 20 miles of track sections are in the good, opportunistic, poor, or critical conditions, and full traffic volume may pass. If any one asset suffers a major degradation, this would limit the total throughput on the rail line, and reduce overall availability. Results also reveal the "at-risk" time periods, where a major degradation condition exists but has not yet been inspected, and so vehicles are passing at an unsafe speed. Finally, the model outputs the expected maintenance volumes, divided between tamping and stoneblowing activities, and the average WO size. Increasing availability performance may be compromised against greater maintenance requirements.

\section{Simulation results and analysis}

Two studies are presented herein. The first set of results establish the lifetime performance expected during fair weather only. The token marking in the weather cycle is omitted for these tests. This reveals the potential long-term benefits found in following opportunistic maintenance policies.

Proceeding, the strategies are carried forward for testing under hot weather disruptions. This focuses on the impact of a single heatwave event on the rail line, initiated once a steady-state geometry condition is reached. These results expose the degree of resilience to periods without maintenance work.

\section{Fair weather simulations}

Table 3 summarises the simulated performance through a 30 year lifetime. These results are averaged for 1,500 iterations, through which result converge in all strategy cases. Even without OM practices, excellent availability is predicted, greater than $96 \%$ of the operational time.

The opportunistic maintenance strategies are all predicted to provide greater availability than the base case, with single asset work orders. Even a minimal geometry range between $\sigma_{\text {opp }}=1.5 \mathrm{~mm}$ and $\sigma_{\text {poor }}=$ $1.9 \mathrm{~mm}$, or narrow search range $r= \pm 5$ provides a benefit to the asset management. This combination gives a small $\mathrm{OM}$ window, and improves the 30-year availability by close to $0.5 \%$. The largest OM window with $\sigma_{o p p}=0.5 \mathrm{~mm}$ and $r= \pm 21$, contributes almost $1.5 \%$ additional availability.

Increasing the availability performance has an in-kind effect on reducing the incidence of major degradation conditions. The non-OM strategy experiences $0.0525 \%$ of the lifetime with a traffic restriction enforced, combining speed restrictions and closures. This equates to approximately 6 days of disruption on the 20 mile line through the 30 year lifetime. The most minimal disruption experienced under $\mathrm{OM}$ is $0.0328 \%$ combined, or less than 4 days over the 30 years. Following the aggressive OM strategy could reduce delays by more than one third, during fair weather.

Similar trends are experienced for the at-risk durations, when the major degradation conditions are not yet detected by inspections. Without OM, vehicles are travelling at unsafe speeds for $3.86 \%$ of the lifetime - approximately two weeks per year - with the

Table 3. Fair weather averaged lifetime performance results

\begin{tabular}{|c|c|c|c|c|c|c|c|c|c|}
\hline \multirow[b]{2}{*}{ Strategy } & \multirow[b]{2}{*}{$\begin{array}{l}\text { Full capacity } \\
\text { time }\end{array}$} & \multicolumn{2}{|c|}{ Speed restrictions } & \multicolumn{2}{|c|}{ Closures } & \multicolumn{2}{|c|}{ Tamping } & \multicolumn{2}{|c|}{ Stoneblowing } \\
\hline & & $\begin{array}{l}\text { Unknown } \\
\text { time }\end{array}$ & $\begin{array}{l}\text { Enforced } \\
\text { time }\end{array}$ & $\begin{array}{l}\text { Unknown } \\
\text { time }\end{array}$ & $\begin{array}{l}\text { Enforced } \\
\text { time }\end{array}$ & $\begin{array}{l}\text { Total } \\
\text { number }\end{array}$ & $\begin{array}{l}\text { Average } \\
\text { WO }\end{array}$ & $\begin{array}{l}\text { Total } \\
\text { number }\end{array}$ & $\begin{array}{l}\text { Average } \\
\text { WO }\end{array}$ \\
\hline Non-OM & $96.0863 \%$ & $3.4682 \%$ & $0.0461 \%$ & $0.3930 \%$ & $0.0064 \%$ & 526.34 & 1.00 & 291.79 & 1.00 \\
\hline$\sigma_{\text {opp }}=0.5, r= \pm 5$ & $96.9652 \%$ & $2.6949 \%$ & $0.0360 \%$ & $0.2992 \%$ & $0.0047 \%$ & 531.51 & 1.47 & 309.85 & 1.37 \\
\hline$\sigma_{o p p}=0.5, r= \pm 9$ & $97.1666 \%$ & $2.5153 \%$ & $0.0330 \%$ & $0.2807 \%$ & $0.0044 \%$ & 547.91 & 1.72 & 312.04 & 1.53 \\
\hline$\sigma_{o p p}=0.5, r= \pm 21$ & $97.5154 \%$ & $2.2063 \%$ & $0.0291 \%$ & $0.2454 \%$ & $0.0037 \%$ & 582.23 & 2.29 & 303.85 & 1.84 \\
\hline$\sigma_{\text {opp }}=1.0, r= \pm 5$ & $96.7730 \%$ & $2.8662 \%$ & $0.0377 \%$ & $0.3182 \%$ & $0.0049 \%$ & 532.95 & 1.37 & 296.08 & 1.27 \\
\hline$\sigma_{\text {opp }}=1.0, r= \pm 9$ & $96.9617 \%$ & $2.6958 \%$ & $0.0355 \%$ & $0.3024 \%$ & $0.0046 \%$ & 546.06 & 1.58 & 295.87 & 1.39 \\
\hline$\sigma_{o p p}=1.0, r= \pm 21$ & $97.2513 \%$ & $2.4477 \%$ & $0.0319 \%$ & $0.2649 \%$ & $0.0042 \%$ & 573.82 & 2.05 & 286.90 & 1.63 \\
\hline$\sigma_{o p p}=1.5, r= \pm 5$ & $96.5205 \%$ & $3.0864 \%$ & $0.0407 \%$ & $0.3472 \%$ & $0.0052 \%$ & 531.55 & 1.26 & 287.55 & 1.17 \\
\hline$\sigma_{o p p}=1.5, r= \pm 9$ & $96.6544 \%$ & $2.9693 \%$ & $0.0387 \%$ & $0.3320 \%$ & $0.0056 \%$ & 542.09 & 1.42 & 284.47 & 1.25 \\
\hline$\sigma_{\text {opp }}=1.5, r= \pm 21$ & $96.9092 \%$ & $2.7445 \%$ & $0.0362 \%$ & $0.3053 \%$ & $0.0046 \%$ & 563.14 & 1.80 & 274.86 & 1.44 \\
\hline
\end{tabular}


expected strong bias for the speed restriction condition. Again, all the OM strategies reduce the risk inherent in the operational management; the small $\mathrm{OM}$ window combination decreases risk to $3.43 \%$ of the lifetime combined, and the greatest OM performance to $2.45 \%$ lifetime risk - less than nine days per year. Whilst availability is an important performance indicator when selecting an asset management strategy, a large reduction in the at-risk durations - up to one third less time at risk - should also feature greatly in the decision making.

Increased availability performance is achieved through greater maintenance delivery. Without opportunistic maintenance strategy, 526 tamping and 292 stoneblowing actions are required over the 30 year lifetime. Across the 160 section rail line, this is equivalent to slightly more than 5 actions per section on average. Of course these are all delivered as single-action work orders, indicated in the average WO size of 1 .

All of the OM strategies call for increased delivery of tamping maintenance. Tamping is more likely to be required at the opportunistic condition, so this preference is expected. The requirement varies between 532 tamping actions for the smaller search ranges, $r= \pm 5$, up to 582 for the largest $\mathrm{OM}$ window with $\sigma_{\text {opp }}=0.5 \mathrm{~mm}$ and $r= \pm 21$. This increase to tamping volume also requires the largest average WO size of 2.3.

Stoneblowing maintenance requirements do not follow the same trend. Increasing the OM search range leads to a decreasing number of maintenance actions. All three scenarios following $\sigma_{o p p}=1.5 \mathrm{~mm}$ call for fewer stoneblowings than the non-OM base level, down to 275 actions following $\sigma_{o p p}=1.5 \mathrm{~mm}$ and $r= \pm 21$. This means an OM strategy can provide a greater availability performance with a lower requirement for stoneblowing, if this is desirable for asset management.

\section{Simulations under hot weather effects}

The hot weather test scenarios follow $\tau_{\text {hot }}=$ $1,2,3,4,5,10,15,20,25,30$ days of heatwave event, though only the final 30 day result is presented in table 5. The full results summary can be found in the

Table 5. Predicted condition and maintenance requirements at the end of a heatwave period

\begin{tabular}{|c|c|c|c|c|c|c|c|}
\hline \multirow[b]{2}{*}{ Scenarios } & \multirow[b]{2}{*}{$\begin{array}{l}\text { Full capacity } \\
\text { probability }\end{array}$} & \multicolumn{2}{|c|}{ Speed restrictions } & \multicolumn{2}{|c|}{ Line closures } & \multirow[b]{2}{*}{$\begin{array}{l}\text { Tamping } \\
\text { number }\end{array}$} & \multirow[b]{2}{*}{$\begin{array}{l}\text { Stoneblowing } \\
\text { number }\end{array}$} \\
\hline & & $\begin{array}{l}\text { Unknown } \\
\text { probability }\end{array}$ & $\begin{array}{l}\text { Enforced } \\
\text { probability }\end{array}$ & $\begin{array}{l}\text { Unknown } \\
\text { probability }\end{array}$ & $\begin{array}{l}\text { Enforced } \\
\text { probability }\end{array}$ & & \\
\hline \multicolumn{8}{|l|}{ Non-OM } \\
\hline Initial & $96.6333 \%$ & $3.1000 \%$ & $0.0000 \%$ & $0.2667 \%$ & $0.0000 \%$ & 0.00 & 0.00 \\
\hline 30 day & $84.2667 \%$ & $5.3333 \%$ & $8.1000 \%$ & $0.5333 \%$ & $1.7667 \%$ & 1.65 & 0.36 \\
\hline \multicolumn{8}{|c|}{ Opportunistic threshold $\sigma_{o p p}=0.5 \mathrm{~mm}$, Search range $r= \pm 5$} \\
\hline Initial & $96.4667 \%$ & $3.1667 \%$ & $0.0000 \%$ & $0.3667 \%$ & $0.0000 \%$ & 0.00 & 0.00 \\
\hline 30 day & $83.8667 \%$ & $5.3000 \%$ & $7.8667 \%$ & $0.9000 \%$ & $2.0667 \%$ & 2.23 & 0.37 \\
\hline \multicolumn{8}{|c|}{ Opportunistic threshold $\sigma_{o p p}=0.5 \mathrm{~mm}$, Search range $r= \pm 9$} \\
\hline Initial & $97.0333 \%$ & $2.6667 \%$ & $0.0000 \%$ & $0.3000 \%$ & $0.0000 \%$ & 0.00 & 0.00 \\
\hline 30 day & $86.0000 \%$ & $4.8000 \%$ & $6.8333 \%$ & $0.7000 \%$ & $1.6667 \%$ & 2.53 & 0.40 \\
\hline \multicolumn{8}{|c|}{ Opportunistic threshold $\sigma_{o p p}=0.5 \mathrm{~mm}$, Search range $r= \pm 21$} \\
\hline Initial & $97.2000 \%$ & $2.6333 \%$ & $0.0000 \%$ & $0.1667 \%$ & $0.0000 \%$ & 0.00 & 0.00 \\
\hline 30 day & $87.4667 \%$ & $4.8000 \%$ & $6.0000 \%$ & $0.5000 \%$ & $1.2333 \%$ & 2.95 & 0.35 \\
\hline \multicolumn{8}{|c|}{ Opportunistic threshold $\sigma_{o p p}=1.0 \mathrm{~mm}$, Search range $r= \pm 5$} \\
\hline Initial & $96.1667 \%$ & $3.7333 \%$ & $0.0000 \%$ & $0.1000 \%$ & $0.0000 \%$ & 0.00 & 0.00 \\
\hline 30 day & $83.6000 \%$ & $5.3333 \%$ & $8.7000 \%$ & $0.5000 \%$ & $1.8667 \%$ & 2.03 & 0.39 \\
\hline \multicolumn{8}{|c|}{ Opportunistic threshold $\sigma_{o p p}=1.0 \mathrm{~mm}$, Search range $r= \pm 9$} \\
\hline Initial & $96.7333 \%$ & $2.8000 \%$ & $0.0000 \%$ & $0.4667 \%$ & $0.0000 \%$ & 0.00 & 0.00 \\
\hline 30 day & $84.1667 \%$ & $6.2000 \%$ & $7.3667 \%$ & $0.5000 \%$ & $1.7667 \%$ & 2.35 & 0.37 \\
\hline \multicolumn{8}{|c|}{ Opportunistic threshold $\sigma_{o p p}=1.0 \mathrm{~mm}$, Search range $r= \pm 21$} \\
\hline Initial & $97.1333 \%$ & $2.5000 \%$ & $0.0000 \%$ & $0.3667 \%$ & $0.0000 \%$ & 0.00 & 0.00 \\
\hline 30 day & $85.8333 \%$ & $4.5333 \%$ & $7.4333 \%$ & $0.5333 \%$ & $1.6667 \%$ & 2.78 & 0.34 \\
\hline \multicolumn{8}{|c|}{ Opportunistic threshold $\sigma_{o p p}=1.5 \mathrm{~mm}$, Search range $r= \pm 5$} \\
\hline Initial & $96.8667 \%$ & $2.8000 \%$ & $0.0000 \%$ & $0.3333 \%$ & $0.0000 \%$ & 0.00 & 0.00 \\
\hline 30 day & $83.7667 \%$ & $6.0000 \%$ & $7.7667 \%$ & $0.7667 \%$ & $1.7000 \%$ & 1.83 & 0.37 \\
\hline \multicolumn{8}{|c|}{ Opportunistic threshold $\sigma_{o p p}=1.5 \mathrm{~mm}$, Search range $r= \pm 9$} \\
\hline Initial & $96.8000 \%$ & $2.7333 \%$ & $0.0000 \%$ & $0.4667 \%$ & $0.0000 \%$ & 0.00 & 0.00 \\
\hline 30 day & $84.2667 \%$ & $5.3000 \%$ & $7.9333 \%$ & $0.7000 \%$ & $1.8000 \%$ & 2.11 & 0.35 \\
\hline \multicolumn{8}{|c|}{ Opportunistic threshold $\sigma_{o p p}=1.5 \mathrm{~mm}$, Search range $r= \pm 21$} \\
\hline Initial & $96.7000 \%$ & $3.1000 \%$ & $0.0000 \%$ & $0.2000 \%$ & $0.0000 \%$ & 0.00 & 0.00 \\
\hline 30 day & $84.6667 \%$ & $5.5000 \%$ & $7.8333 \%$ & $0.6333 \%$ & $1.3667 \%$ & 2.37 & 0.34 \\
\hline
\end{tabular}


appendix. Resilience is exhibited by retaining high availability through the disruption period.

In the scenario without opportunistic maintenance practice, availability falls from $96 \%$ to $84 \%$ through 30 days of heatwave. Greater availability is predicted at the end of the long heatwave periods by four of the OM strategies; combination $\sigma_{o p p}=0.5 \mathrm{~mm}, r= \pm 9$, and all three thresholds when paired with search range $r= \pm 21$. By providing high initial availability, these strategies are able to provide performance resilience through the maintenance-free period.

In contrast, those cases which do not exhibit good resilience reveal the negative aspect of an increased tamping maintenance regime. As has been identified, a history of tamping actions will lead to greater rail geometry degradation rates. Though a rail section may enjoy frequent opportunistic maintenance in fair weather, the increased degradation rate is detrimental during a heatwave disruption. This means availability performance drops-off at a higher rate than the non-OM base level.

The heatwave scenarios predict greater levels of major degradation conditions as a result of the maintenance inhibition; no urgent maintenance can be completed, and so the probability is increased that a traffic restriction is enforced. Most of the OM parameter combinations exhibit a reduced probability of a speed restriction or line closure enforcement. The strategy with $\sigma_{o p p}=1.0 \mathrm{~mm}, r= \pm 5$ is however poorly optimised, predicting a greater chance of traffic restriction than no $\mathrm{OM}$ at all.

The maintenance volumes as reported in table 5 represent the accumulated demand through the heatwave inhibition. That is, the number of maintenance actions that have come to schedule and are expected to be completed as soon as the hot weather has broken. All of the OM strategies demand more maintenance than the base level; the non-OM strategy calls for 2 maintenance actions at the end of 30 days, between the two maintenance types, whilst the highly performing combination $\sigma_{o p p}=0.5 \mathrm{~mm}, r= \pm 21$ demands 3.3 total actions. This highlights the compromise that must be met between increasing fair weather performance and growing maintenance volumes during maintenance-free periods.

\section{Conclusions}

The CPN presented in this study models the degradation, inspection, and maintenance planning and delivery for a railway route comprising of a series of track sections. The model utilises colour sets to distinguish the multiple individual assets in a compact graphical form. The CPN is successfully capable of simulating the entire rail line with unique degradation progression for each individual track section, and with management decisions made for the combined system. By considering the entire route - in contrast to single track sections in isolation - effective management strategies are explored, including interdependencies permitted by local adjacency.
The novel search transition function finds asset tokens that match the defined criteria, namely locations within a given adjacency range. This enables opportunistic maintenance practice, dynamically grouping interventions for neighbouring track sections into a single work order. Comparing simulation results for the different $\mathrm{OM}$ strategies gives an indication of their effectiveness in delivering a safe and resilient network.

The initial results study compares 10 asset management strategies acting to provide high availability across a 30 year lifetime simulation. By including the opportunistic maintenance search function, asset management may provide a highly available, reduced risk rail line, though at the expense of increased maintenance requirements. The combination of a broad opportunistic maintenance condition threshold and a wide-ranging adjacency search distance predict the greatest availability performance, though even a small OM window is able to provide a benefit over the non-opportunistic approach.

Hot weather continues to be a significant problem for railway asset management, costing up to $£ 15 \mathrm{~m}$ in delays and cancellations in the UK during a modern summer ${ }^{34}$. In the CPN model, hot weather is introduced as an inhibiting factor for completing a work order, producing an extended maintenance-free time period.

Resilience to the hot weather is exhibited in providing a high availability throughout the disruption time. As before, a broad opportunistic threshold and wide search range are a strategy combination that is predicted to provide high availability performance through a long maintenance-free heatwave period. This is however at the compromise of an increased simultaneous maintenance demand at the end of the hot weather, from an average 2 actions without $\mathrm{OM}$ to 3.3 in the greatest case. Though these numbers are small for the modelled 20 mile rail line, the proportional increase is not insignificant for asset management planning across a region.

Opportunistic maintenance was not beneficial in all test cases. Poorly optimised parameter combinations exhibit greater performance loss and high probability of a traffic restriction enforcement than the case with no $\mathrm{OM}$ at all. This is a penalty of an increased tamping history on the line, which is known to induce worse geometry degradation rates thereafter.

The results of this study suggest that asset managers should be motivated to deliver opportunistic maintenance strategies to support a rail network with good resilience to disrupting weather events. Grouping assets together on a broad adjacency search range will demand larger maintenance work orders to be completed, though ensure that assets are in better overall condition. Excellent steady-state condition is a great contributor to ensuring availability performance through a maintenance-free period. It may also be beneficial to follow different asset management strategies during seasons of fair weather and heatwaves.

This model contributes to the suite of asset management tools that are used by the rail industry. Simulation results can provide guidance on maintenance 
policies, and a prediction of performance that is forecast by empirical modelling. The strategies explored in this study focus on the model parameters related to opportunistic maintenance practice. Other features of the asset management policy can be parametrised for testing, as has been explored in a previous publication ${ }^{1}$.

One limitation of this model is the omission of a cost analysis of varying maintenance demands, and the penalties associated with network delays. This would be important for appraising the compromise in achieving increased availability performance whilst delivering greater maintenance volume. Further work should implement costing for all works and delay estimates, and an optimisation study.

The CPN approach lends itself well to the task of modelling railway asset management practice. This has similarly been demonstrated in existing publications of traditional Petri net models. In the current format, the model in this study could be parameterised for other asset management processes, where a combination of corrective, preventive, and opportunistic maintenance is to be delivered. Further, multiple degradation processes could be studied in the same asset management strategy, by introducing condition states in parallel to the track geometry. This could represent other measures of track geometry, or different assets that are in the same environment. The Coloured Petri Net modelling approach has sufficient flexibility to be applied to a range of engineering processes of complex behaviour and interaction.

\section{Acknowledgements}

John Andrews is the Royal Academy of Engineering and Network Rail Professor of Infrastructure Asset Management, and Ben Davies is conducting a research project funded by Network Rail. They gratefully acknowledge the support from Network Rail.

\section{References}

1. Ben Davies and John Andrews. The impact of summer heatwaves on railway track geometry maintenance. Proceedings of the Institution of Mechanical Engineers, Part F: Journal of Rail and Rapid Transit, 2021.

2. Y. J. Zhang, M. Murray, and Luis Ferreira. Modelling rail track performance: An integrated approach. Proceedings of the Institution of Civil Engineers: Transport, 141(4):187-194, 2000.

3. Iman Soleimanmeigouni, Alireza Ahmadi, and Uday Kumar. Track geometry degradation and maintenance modelling: A review. Proceedings of the Institution of Mechanical Engineers, Part F: Journal of Rail and Rapid Transit, 232(1):73-102, 2018.

4. Darren Prescott and John Andrews. Modelling maintenance in railway infrastructure management. Proceedings - Annual Reliability and Maintainability Symposium, pages 1-6, jan 2013.

5. Bernhard Lichtberger. Track Compendium. Hamburg, Eurailpress Tetzlaff-Hestra GmbH \& Co. Publ, pages 1$192,2005$.
6. Peter Musgrave. Track bed total route evaluation for track renewals and asset management "a Network Rail perspective". Construction and Building Materials, 92:2-8, sep 2015.

7. Matthew Audley and John Andrews. The effects of tamping on railway track geometry degradation. Proceedings of the Institution of Mechanical Engineers, Part F: Journal of Rail and Rapid Transit, 227(4):376391, 2013.

8. P Claisse and C Calla. Rail ballast: Conclusions from a historical perspective. Proceedings of the Institution of Civil Engineers: Transport, 159(2):69-74, 2006.

9. Network Rail. NR/L2/TRK/3011 Continuous Welded Rail (CWR) Track. Technical report, 2012.

10. A. Ramos Andrade and P. Fonseca Teixeira. Biobjective optimization model for maintenance and renewal decisions related to rail track geometry. Transportation Research Record, (2261):163-170, 2011.

11. G. Budai, D. Huisman, and R. Dekker. Scheduling preventive railway maintenance activities. Journal of the Operational Research Society, 57(9):1035-1044, 2006.

12. M. Sol-Sánchez, F. Moreno-Navarro, and M. C. RubioGámez. Analysis of ballast tamping and stone-blowing processes on railway track behaviour: The influence of using USPs. Geotechnique, 66(6):481-489, 2016.

13. Darren Prescott and John Andrews. A track ballast maintenance and inspection model for a rail network. Proceedings of the Institution of Mechanical Engineers, Part O: Journal of Risk and Reliability, 227(3):251-266, 2013.

14. Jianmin Zhao, A. H.C. Chan, A. B. Stirling, and K. B. Madelin. Optimizing policies of railway ballast tamping and renewal. Transportation Research Record, 1943(1943):50-56, 2006.

15. T. R. Sussmann, W. Ebersöhn, and E. T. Selig. Fundamental nonlinear track load-deflection behavior for condition evaluation. Transportation Research Record, 3(1742):61-67, 2001.

16. Qing Chang, Jun Ni, Pulak Bandyopadhyay, Stephan Biller, and Guoxian Xiao. Maintenance staffing management. Journal of Intelligent Manufacturing, 18(3):351-360, 2007.

17. Jørn Vatn. Maintenance in the Rail Industry. Springer Series in Reliability Engineering, 8:509-531, 2008.

18. David J. Sherwin. Age-based opportunity maintenance. Journal of Quality in Maintenance Engineering, 5(3):221-235, 1999.

19. K Dobney, C. J. Baker, A. D. Quinn, and L. Chapman. Quantifying the effects of high summer temperatures due to climate change on buckling and rail related delays in south-east United Kingdom. Meteorological Applications, 16(2):245-251, jun 2009.

20. Tao Zhang, John Andrews, and Rui Wang. Optimal scheduling of track maintenance on a railway network. Quality and Reliability Engineering International, 29(2):285-297, 2013.

21. Scott Simson, Luis Ferreira, and Martin Murray. Modelling track maintenance planning using simulation. Proceedings of the Australian Society for Operations Research 15th National conference, pages 1159-1172, 1999. 
22. Mauro Dell'orco, Michele Ottomanelli, Leonardo Caggiani, and Domenico Sassanelli. New Decision Support System for Optimization of Rail Track Maintenance Planning Based on Adaptive Neurofuzzy Inference System. Transportation Research Record: Journal of the Transportation Research Board, 2043:4954, 2008.

23. Tatsuo Oyama and Masashi Miwa. Mathematical modeling analyses for obtaining an optimal railway track maintenance schedule. Japan Journal of Industrial and Applied Mathematics, 23(2):207-224, 2006.

24. Rui Santos, Paulo Fonseca Teixeira, and Antonio Pais Antunes. Planning and scheduling efficient heavy rail track maintenance through a Decision Rules Model. Research in Transportation Economics, 54:20-32, 2015.

25. Stephen M. Famurewa, Tao Xin, Matti Rantatalo, and Uday Kumar. Optimisation of maintenance track possession time: A tamping case study. Proceedings of the Institution of Mechanical Engineers, Part F: Journal of Rail and Rapid Transit, 229(1):12-22, 2015.

26. Hamid Khajehei, Mohammad Haddadzade, Alireza Ahmadi, Iman Soleimanmeigouni, and Arne Nissen. Optimal opportunistic tamping scheduling for railway track geometry. Structure and Infrastructure Engineering, 0(0):1-16, 2020.

27. J. Zhao, A. H.C. Chan, and M. P.N. Burrow. A geneticalgorithm-based approach for scheduling the renewal of railway track components. Proceedings of the Institution of Mechanical Engineers, Part F: Journal of Rail and Rapid Transit, 223(6):533-541, 2009.

28. Fan Peng, Seungmo Kang, Xiaopeng Li, Yanfeng Ouyang, Kamalesh Somani, and Dharma Acharya. A Heuristic Approach to the Railroad Track Maintenance Scheduling Problem. Computer-Aided Civil and Infrastructure Engineering, 26(2):129-145, 2011.

29. John Andrews. A modelling approach to railway track asset management. Proceedings of the Institution of Mechanical Engineers, Part F: Journal of Rail and Rapid Transit, 227(1):56-73, jan 2013.

30. Matthew Audley and John Andrews. A PetriNet Modelling Approach to Rail Track Geometry Maintenance and Inspection. In Proceedings of the 20th Advances in Risk and Reliability Technology Symposium, pages 230-243, 2013.

31. Jack Litherland and John Andrews. A Petri Net Methodology for Modelling the Maintenance of Railway Route Sections. Proceedings of the 29th European Safety and Reliability Conference, 2019.

32. Claudia Fecarotti and John Andrews. A mathematical programming approach to railway network asset management. Safety and Reliability - Safe Societies in a Changing World, pages 839-847, 2018.

33. Emma Ferranti, Lee Chapman, Caroline Lowe, Steve McCulloch, David Jaroszweski, and Andrew Quinn. Heat-Related Failures on Southeast England's Railway Network: Insights and Implications for Heat Risk Management. Weather, Climate, and Society, 8(2):177191,2016

34. K Dobney, C J Baker, L Chapman, and A D Quinn. The future cost to the United Kingdom's railway network of heat-related delays and buckles caused by the predicted increase in high summer temperatures owing to climate change. Proceedings of the Institution of Mechanical Engineers, Part F: Journal of Rail and Rapid Transit, 224(1):25-34, 2010.

35. Kurt Jensen. Coloured Petri Nets: Basic concepts, analysis methods and practical use. 2 edition, 1997.

36. Network Rail. Track treatment fleet - Network Rail. Technical report, www.networkrail.co.uk/running-therailway/looking-after-the-railway/our-fleet-machinesand-vehicles/track-treatment-fleet/, 2020.

37. Erika J. Palin, Hazel E. Thornton, Camilla T. Mathison, Rachel E. McCarthy, Robin T. Clark, and John Dora. Future projections of temperature-related climate change impacts on the railway network of Great Britain. Climatic Change, 120(1-2):71-93, sep 2013. 